\title{
Di-Higgs boson peaks and top valleys: Interference effects in Higgs sector extensions
}

\author{
Philipp Basler, ${ }^{1, *}$ Sally Dawson, ${ }^{2, \dagger}$ Christoph Englert, ${ }^{3, *}$ and Margarete Mühlleitner, ${ }^{1, \S}$ \\ ${ }^{1}$ Institute for Theoretical Physics, Karlsruhe Institute of Technology, 76128 Karlsruhe, Germany \\ ${ }^{2}$ Department of Physics, Brookhaven National Laboratory, Upton, New York 11973, USA \\ ${ }^{3}$ SUPA, School of Physics and Astronomy, University of Glasgow, Glasgow G12 8QQ, United Kingdom
}

(Received 7 October 2019; revised manuscript received 13 December 2019; published 28 January 2020)

In models with extended scalars and $C P$ violation, resonance searches in double Higgs final states stand in competition with related searches in top quark final states as optimal channels for the discovery of beyond the Standard Model (BSM) physics. This complementarity is particularly relevant for benchmark scenarios that aim to highlight multi-Higgs production as a standard candle for the study of BSM phenomena. In this paper, we compare interference effects in $t \bar{t}$ final states with correlated phenomena in double Higgs production in the complex singlet and the complex two-Higgs-doublet models. Our results indicate that the BSM discovery potential in di-Higgs searches can be underestimated in comparison to $t \bar{t}$ resonance searches. Top pair final states are typically suppressed due to destructive signal-background interference, while $h h$ final states can be enhanced due to signal-signal interference. For parameter choices where the two heavy Higgs resonances are well separated in mass, top final states are suppressed relative to the naive signal expectation, while estimates of the production cross section times branching ratio remain accurate at the $\mathcal{O}(10 \%)$ level for double Higgs final states.

DOI: 10.1103/PhysRevD.101.015019

\section{INTRODUCTION}

The search for new physics beyond the Standard Model remains a priority of the Large Hadron Collider (LHC) phenomenology program. Although model-independent search strategies are gaining momentum, concrete and well-motivated UV scenarios still provide vital information on why new physics has not been observed so far. Recent investigations [1-4] highlight the point that the nonobservation of new physics can be reconciled with "standard" Higgs sector extensions far away from their decoupling limits. This is possible when the non-Standard Model (non-SM) degrees of freedom have suppressed production cross sections, or when they are hidden in channels that are experimentally difficult to observe. Particularly relevant in this context are di-Higgs final states that might act as the main discovery channel for BSM physics in such an instance.

The largest production channel for electroweak scalars that have significant top couplings proceeds through gluon

\footnotetext{
"philipp.basler@kit.edu

dawson@bnl.gov

*christoph.englert@glasgow.ac.uk

§milada.muehlleitner@kit.edu
}

Published by the American Physical Society under the terms of the Creative Commons Attribution 4.0 International license. Further distribution of this work must maintain attribution to the author(s) and the published article's title, journal citation, and DOI. Funded by SCOAP. fusion at the LHC; see e.g., [5]. This directly motivates resonance searches in top final states if they are kinematically accessible. It is known that resonance searches in top pairs are particularly vulnerable to large interference effects [6-19] that can have a significant impact on the formulation of exclusion constraints [20-23]. It is therefore entirely possible that a new (possibly gauge-phobic) scalar is not visible as an isolated $t \bar{t}$ resonance. Under these circumstances, multi-Higgs production becomes a crucial tool for BSM discovery.

It is the purpose of this paper to quantitatively compare $t \bar{t}$ and $h h$ resonance searches for models where we would expect top production to play the leading role in a new physics discovery. This is particularly highlighted in the complex singlet (CxSM) and $C P$-violating two-Higgsdoublet models (C2HDM). Building on previous insights [2], we show that in the relevant regions of the C2HDM interference effects can lead to misleading sensitivity estimates. In particular, the sensitivity from di-Higgs final states [24] can be underestimated relative to top pair production; i.e., destructive interference effects in $t \bar{t}$ searches correlate with constructive interference in $h h$ searches in parameter regions of the C2HDM that are allowed in the light of current LHC searches. We contrast these findings against results obtained for the CxSM.

This work is organized as follows. In Sec. II, we briefly review the CxSM and the C2HDM that we consider in this work. We provide details of our calculation with results in 
Sec. III B, where we also present a number of benchmark points that highlight the phenomenology. We conclude in Sec. IV.

\section{MODELS AND SCANS}

\section{A. The CxSM}

The CxSM is based upon the extension of the SM by a complex scalar field [25-33]. The CxSM potential with a softly broken global $U(1)$ symmetry is given by

$$
\begin{aligned}
V= & \frac{m^{2}}{2} H^{\dagger} H+\frac{\lambda}{4}\left(H^{\dagger} H\right)^{2}+\frac{\delta_{2}}{2} H^{\dagger} H|\mathbb{S}|^{2}+\frac{b_{2}}{2}|\mathbb{S}|^{2} \\
& +\frac{d_{2}}{4}|\mathbb{S}|^{4}+\left(\frac{b_{1}}{4} \mathbb{S}^{2}+a_{1} \mathbb{S}+\text { c.c. }\right),
\end{aligned}
$$

with

$$
\mathbb{S}=S+i A
$$

being a hypercharge zero scalar field and the soft breaking terms being written in parenthesis. After electroweak symmetry breaking the fields can be written as

$$
H=\frac{1}{\sqrt{2}}\left(\begin{array}{c}
G^{+} \\
v+h+i G^{0}
\end{array}\right)
$$

and

$$
\mathbb{S}=\frac{1}{\sqrt{2}}\left[v_{S}+s+i\left(v_{A}+a\right)\right],
$$

where $v \approx 246 \mathrm{GeV}$ is the $\mathrm{SM}$ vacuum expectation value (VEV) of the $h$ field and $v_{S}$ and $v_{A}$ are the VEVs of the real and imaginary parts of the complex singlet field, respectively. The Hermiticity of the potential implies that all parameters are real, except for the soft breaking terms. We also impose invariance under $\mathbb{S} \rightarrow \mathbb{S}^{*}$ (or $A \rightarrow-A$ ) so that $a_{1}$ and $b_{1}$ are real. This implies that the theory is described by seven independent parameters. The model can be studied by treating the real and imaginary components of the complex singlet as independent fields, which implies that the model is equivalent to one with two real singlets and has no $C P$ violation. For our investigation we choose to work in the broken phase where all three VEVs are nonzero, as this phase implies mixing between all three $C P$-even scalars. Their mass eigenstates $H_{i}(i=1,2,3)$ are obtained from the gauge eigenstates through the rotation matrix $R$ parametrized as

$R=\left(\begin{array}{ccc}c_{1} c_{2} & s_{1} c_{2} & s_{2} \\ -\left(c_{1} s_{2} s_{3}+s_{1} c_{3}\right) & c_{1} c_{3}-s_{1} s_{2} s_{3} & c_{2} s_{3} \\ -c_{1} s_{2} c_{3}+s_{1} s_{3} & -\left(c_{1} s_{3}+s_{1} s_{2} c_{3}\right) & c_{2} c_{3}\end{array}\right)$,

where we have introduced the shorthand notation $s_{i} \equiv$ $\sin \alpha_{i}$ and $c_{i} \equiv \cos \alpha_{i}$. Without loss of generality we vary the angles in the range

$$
-\frac{\pi}{2} \leq \alpha_{i}<\frac{\pi}{2} .
$$

The masses of the neutral Higgs bosons are ordered as $m_{H_{1}} \leq m_{H_{2}} \leq m_{H_{3}}$. As input parameters we choose the set

$\alpha_{1}, \quad \alpha_{2}, \quad \alpha_{3}, \quad v, \quad v_{S}, \quad m_{H_{1}}$ and $m_{H_{3}}$.

The remaining parameters are determined internally in Scanners [27,34], with which we perform our scan in the parameter space of the model, taking into account the minimum conditions on the vacuum.

\section{B. The CxSM scan}

In order to find viable points in the parameter space of the CxSM that are compatible with the relevant theoretical and experimental constraints, we performed a scan using the program ScannerS. The program checks for theoretical constraints such as the requirement of the potential to be bounded from below, the chosen vacuum to be a global minimum and perturbative unitarity to be fulfilled. We furthermore require the mass of the lightest Higgs boson, identified with the SM-like one and denoted by $h$, to be $m_{h}=125.09 \mathrm{GeV}$ [35]. Compatibility with the electroweak precision data is ensured by applying a 95\% C.L. exclusion limit from the electroweak precision observables $S, T$ and $U$ [36,37]; see [38] for further details. Compatibility with the exclusion limits from the collider data on Higgs observables at $95 \%$ C.L. has been checked by using HiggsBounds5.2.0 [39-41] and compatibility with the Higgs rates was verified by using HiggsSignals2.2.1 [42]. The necessary production cross sections were obtained from ScannerS which uses results from SusHi1.6.1 [43,44]. The required branching ratios to compute the signal strength were computed with SHDECAY [30] which is based on the implementation of the CxSM and also the real singlet extension of the SM $(\mathrm{RxSM})$ both in their symmetric and broken phases in HDECAY $[45,46]$.

The SM input parameters are chosen as $[47,48]$

$$
\begin{array}{rlrl}
\alpha\left(M_{Z}\right) & =1 / 127.92, & \alpha_{s}^{\overline{\mathrm{MS}}}\left(M_{Z}\right)=0.118, \\
M_{Z} & =91.187 \mathrm{GeV}, \quad M_{W}=80.358 \mathrm{GeV}, \\
m_{t} & =172.5 \mathrm{GeV}, \quad m_{b}^{\overline{\mathrm{MS}}}\left(m_{b}^{\overline{\mathrm{MS}}}\right)=4.18 \mathrm{GeV}, \\
m_{\tau} & =1.777 \mathrm{GeV} .
\end{array}
$$

The remaining light quark and lepton masses have been set to $[47,48]$

$$
\begin{aligned}
& m_{e}=0.5110 \mathrm{MeV}, \quad m_{\mu}=105.66 \mathrm{MeV}, \\
& m_{u}=100 \mathrm{MeV}, \quad m_{d}=100 \mathrm{MeV}, \\
& m_{s}=100 \mathrm{MeV} .
\end{aligned}
$$


Our sample points were generated with the input parameters listed in Eqs. (7)-(9). Identifying the lightest Higgs boson with the SM-like Higgs boson $h$, the remaining ones are restricted to the mass range

$$
125.09 \mathrm{GeV}<m_{H_{i}} \leq 1000 \mathrm{GeV}, \quad H_{i} \neq h .
$$

The VEVs $v_{A}$ and $v_{S}$ are varied in the range ${ }^{1}$

$$
1 \mathrm{GeV} \leq v_{A}, \quad v_{S}<1.0 \mathrm{TeV},
$$

and the mixing angles as in Eq. (6). All input parameters except for the mixing angles were generated randomly and uniformly in the ranges specified above. The mixing angles on the other hand were extracted from the mixing matrix elements of $R$ defined in Eq. (5), that were generated uniformly. Through this procedure the couplings to fermions and gauge bosons are distributed uniformly.

We also checked if the parameters of the final data set induce a strong first order phase transition, which is a necessary condition for successful baryogenesis [49-51], by using the $\mathrm{C}++$ code BSMPT [52]. We found that none of the benchmark points satisfies a strong first order phase transition.

\section{The C2HDM}

By adding a second $S U(2)_{L}$ Higgs doublet to the SM Higgs sector we obtain the two-Higgs-doublet model (2HDM) [53-55]. Imposing a $\mathbb{Z}_{2}$ symmetry, under which the two $S U(2)_{L}$ doublets $\Phi_{j}(j=1,2)$ transform as $\Phi_{1} \rightarrow$ $\Phi_{1}$ and $\Phi_{2} \rightarrow-\Phi_{2}$, the Higgs potential of a general 2HDM with the $\mathbb{Z}_{2}$ symmetry softly broken can be cast into the form

$$
\begin{aligned}
V= & m_{11}^{2}\left|\Phi_{1}\right|^{2}+m_{22}^{2}\left|\Phi_{2}\right|^{2} \\
& -\left(m_{12}^{2} \Phi_{1}^{\dagger} \Phi_{2}+\text { H.c. }\right)+\frac{\lambda_{1}}{2}\left(\Phi_{1}^{\dagger} \Phi_{1}\right)^{2} \\
& +\frac{\lambda_{2}}{2}\left(\Phi_{2}^{\dagger} \Phi_{2}\right)^{2}+\lambda_{3}\left(\Phi_{1}^{\dagger} \Phi_{1}\right)\left(\Phi_{2}^{\dagger} \Phi_{2}\right) \\
& +\lambda_{4}\left(\Phi_{1}^{\dagger} \Phi_{2}\right)\left(\Phi_{2}^{\dagger} \Phi_{1}\right)+\left[\frac{\lambda_{5}}{2}\left(\Phi_{1}^{\dagger} \Phi_{2}\right)^{2}+\text { H.c. }\right] .
\end{aligned}
$$

The $\mathbb{Z}_{2}$ symmetry is extended to the fermion sector, thereby guaranteeing the absence of flavor-changing neutral currents. The $\mathbb{Z}_{2}$ charge assignments can be distributed such that we obtain four phenomenologically different $2 \mathrm{HDM}$ types summarized in Table I. For the Higgs potential to be Hermitian, all parameters must be real, with the exception of $\lambda_{5}$ and $m_{12}^{2}$. We obtain the complex or $C P$-violating 2HDM [56] if they have different unrelated complex

\footnotetext{
${ }^{1}$ Note that $v_{A}$ is not varied as an independent input parameter; we solely make sure that by our choice of input parameters $v_{A}$ lies in the range defined in Eq. (11).
}

TABLE I. The four Yukawa types of the softly broken $\mathbb{Z}_{2}$-symmetric $2 \mathrm{HDM}$, defined by the Higgs doublet that couples to each kind of fermion.

\begin{tabular}{lccc}
\hline \hline & $u$-type & $d$-type & Leptons \\
\hline Type I (T1) & $\Phi_{2}$ & $\Phi_{2}$ & $\Phi_{2}$ \\
Type II (T2) & $\Phi_{2}$ & $\Phi_{1}$ & $\Phi_{1}$ \\
Lepton-specific & $\Phi_{2}$ & $\Phi_{2}$ & $\Phi_{1}$ \\
Flipped & $\Phi_{2}$ & $\Phi_{1}$ & $\Phi_{2}$ \\
\hline \hline
\end{tabular}

phases. In the following, we adopt the conventions of [57] for the description of the C2HDM. Also, the phases of the VEVs of the neutral components of the two Higgs doublets after electroweak symmetry breaking (EWSB) can in principle be complex in the C2HDM. Without loss of generality we set them to zero, as they can be removed by a basis change [56]. Expanding the Higgs doublets $\Phi_{j}$ around their respective VEVs $v_{j}(j=1,2)$ after EWSB, they can be written as

$$
\Phi_{1}=\left(\begin{array}{c}
\phi_{1}^{+} \\
\frac{v_{1}+\rho_{1}+i \eta_{1}}{\sqrt{2}}
\end{array}\right) \quad \text { and } \quad \Phi_{2}=\left(\begin{array}{c}
\phi_{2}^{+} \\
\frac{v_{2}+\rho_{2}+i \eta_{2}}{\sqrt{2}}
\end{array}\right),
$$

where the $\phi_{j}^{+}$denote the complex charged fields, and $\rho_{j}$ and $\eta_{j}$ the neutral $C P$-even and $C P$-odd fields, respectively. The VEVs are related to the SM VEV $v \approx 246 \mathrm{GeV}$ through $v_{1}^{2}+v_{2}^{2}=v^{2}$, and their ratio is parametrized by the mixing angle $\beta$,

$$
\tan \beta \equiv t_{\beta}=\frac{v_{2}}{v_{1}}
$$

The mass parameters $m_{11}^{2}$ and $m_{22}^{2}$ of the Higgs potential can be eliminated in favor of $v_{1}$ and $v_{2}$ by exploiting the minimum conditions of the potential that require that its minimum be given by $\left\langle\Phi_{j}\right\rangle=\left(0, v_{j} / \sqrt{2}\right)^{T}$. They also relate the imaginary parts of $m_{12}^{2}$ and $\lambda_{5}$ and thus fix one of the ten Higgs potential parameters. Applying the orthogonal rotation matrix $R$ to the neutral components of the interaction basis, $\rho_{1,2}$ and $\rho_{3} \equiv(1 / \sqrt{2})\left(-\sin \beta \eta_{1}+\right.$ $\left.\cos \beta \eta_{2}\right)$, we obtain the neutral Higgs mass eigenstates $H_{i}$ $(i=1,2,3)$,

$$
\left(\begin{array}{l}
H_{1} \\
H_{2} \\
H_{3}
\end{array}\right)=R\left(\begin{array}{c}
\rho_{1} \\
\rho_{2} \\
\rho_{3}
\end{array}\right)
$$

Note that the field $\rho_{3}$ is equal to the $C P$-odd component of the second Higgs doublet in the Higgs basis [58,59]. The matrix $R$ diagonalizes the mass matrix $\mathcal{M}$ of the neutral states,

$$
R \mathcal{M}^{2} R^{T}=\operatorname{diag}\left(m_{H_{1}}^{2}, m_{H_{2}}^{2}, m_{H_{3}}^{2}\right) .
$$


The $m_{H_{i}}$ denote the masses of the neutral Higgs bosons and are ordered by ascending mass, $m_{H_{1}} \leq m_{H_{2}} \leq m_{H_{3}}$. Having a mixing of three Higgs states as in the CxSM the mixing matrix $R$ can be parametrized in terms of the mixing angles $\alpha_{i}$ in the same way as in the CxSM; cf. Eq. (5). The Higgs sector of the C2HDM can be described by nine independent parameters, that we choose to be [60]

$$
\begin{aligned}
& v, \quad t_{\beta}, \quad \alpha_{1,2,3}, \quad m_{H_{i}}, \\
& m_{H_{j}}, \quad m_{H^{ \pm}}, \quad \operatorname{Re}\left(m_{12}^{2}\right) .
\end{aligned}
$$

Here $m_{H_{i}}$ and $m_{H_{j}}$ denote any of the three neutral Higgs boson masses, while the third mass is not independent and is calculated from the other parameters [60]. The triple Higgs couplings are found from the potential,

$$
\begin{aligned}
V= & \frac{1}{3 !} \sum_{i=1}^{3} \lambda_{H_{i} H_{i} H_{i}} H_{i}^{3}+\frac{1}{2} \sum_{i=1}^{3} \sum_{j=i+1}^{3} \lambda_{H_{i} H_{i} H_{j}} H_{i}^{2} H_{j} \\
& +\lambda_{H_{1} H_{2} H_{3}} H_{1} H_{2} H_{3} .
\end{aligned}
$$

Further details, and in particular all Higgs couplings of the C2HDM, can be found in [61]. ${ }^{2}$

\section{The C2HDM scan}

In this work, for simplicity we only consider the C2HDM type 1 (T1) and type 2 (T2), motivated by the fact that they cover to a large extent the phenomenological effects to be expected in the C2HDM. In order to find valid C2DHM points for our investigations, and to define benchmark points $^{3}$ that have not been excluded yet, ${ }^{4}$ we use Scanners to perform a scan in the C2HDM parameter space. As in the CxSM, Scanners checks for the theoretical constraints on the C2HDM Higgs potential, and it uses the tree-level discriminant of [64] to enforce the electroweak vacuum to be the global minimum of the tree-level Higgs potential. We require the mass of the lightest Higgs boson, that is identified with the SM-like one and denoted by $h$, to be $m_{h}=125.09 \mathrm{GeV}$ [35]. In Table II we summarize the ranges of the other scan parameters. Note that the third neutral Higgs boson mass $m_{H_{j} \neq H_{i}, h}$ is calculated from the other input values and forced to lie in the interval given in Table II. In order to circumvent degenerate Higgs signals, we additionally impose $m_{H_{i, j} \neq h}$ to be $5 \mathrm{GeV}$ away from $125 \mathrm{GeV}$. The $\mathrm{SM}$ input parameters are chosen as in the scan for the CxSM. In our scan we neglect parameter points with $\operatorname{Re}\left(m_{12}^{2}\right)<0$, as they are extremely rare. We check all

\footnotetext{
${ }^{2}$ The trilinear Higgs couplings, which are quite complicated, can also be found analytically at http://porthos.tecnico.ulisboa.pt/ arXiv/C2HDM.

${ }^{3}$ For benchmarks for double Higgs production in the 2HDM, see e.g., Refs. [62,63].

${ }^{4}$ See Ref. [2] for our recently proposed benchmark points for C2HDM di-Higgs production.
}

TABLE II. C2HDM scan. All parameters are varied independently between the given minimum and maximum values. The two minimum values of the charged Higgs mass range refer to the scan in the C2HDM T1 and T2, respectively. For more details, see text.

\begin{tabular}{cccccc}
\hline \hline & $t_{\beta}$ & $\alpha_{1,2,3}$ & $\operatorname{Re}\left(m_{12}^{2}\right)\left(\mathrm{TeV}^{2}\right)$ & $m_{H^{ \pm}}(\mathrm{TeV})$ & $m_{H_{i, j} \neq h}(\mathrm{TeV})$ \\
\hline $\operatorname{Min}$ & 0.8 & $-\frac{\pi}{2}$ & 0 & $0.15 / 0.59$ & 0.125 \\
$\operatorname{Max}$ & 20 & $\frac{\pi}{2}$ & 0.5 & 1.5 & 1.5 \\
\hline \hline
\end{tabular}

parameter points at the $2 \sigma$ exclusion level in the $m_{H^{ \pm}}-\tan \beta$ plane for compatibility with the flavor constraints on $R_{b}$ $[65,66]$ and $B \rightarrow X_{s} \gamma$ [66-70]. Applying the results of [70] we require $m_{H^{ \pm}}$to be above $590 \mathrm{GeV}$ in the C2HDM T2. In the C2HDM T1, on the other hand, the bound is much weaker and depends more strongly on $\tan \beta$. Our retained parameter points are put in agreement with the electroweak precision data by demanding $2 \sigma$ compatibility with the SM fit [71] of the oblique parameters $S, T$ and $U$, including the full correlation among the three parameters. The necessary $2 \mathrm{HDM}$ formulas are given in $[55,72]$. For the check of the compatibility with the Higgs data we proceeded as in the CxSM, with the difference that we obtained the necessary branching ratios from the C2HDM implementation C2HDM_HDECAY [61] in HDECAY [45,46]. Further details can be found in $[31,61]$.

Since we work in the C2HDM, we also have to check for agreement with the measurements of the electric dipole moment (EDM), with the strongest constraint originating from the electron EDM [73]. We take the experimental limit given by the ACME Collaboration [74]. As shown explicitly in Figs. 5 and 6 of Ref. [75], the limits from the neutron EDM are weaker than those from the electron EDM. Like for the $\mathrm{CxSM}$ we also checked if the final scenarios induce a strong first order phase transition [52,76]. Also here we found that for none of them is this the case.

\section{INTERFERENCE EFFECTS: TOP VS DI-HIGGS FINAL STATES}

\section{A. Setup}

Based on the scan detailed in Sec. II, we implement the $p p \rightarrow H_{i} \rightarrow t \bar{t}$ and $p p \rightarrow H_{i} \rightarrow h h$ resonant amplitudes into Vbfnlo [77-80], where $H_{i}$ denotes any of the non-SMlike heavy Higgs bosons of the CxSM or C2HDM, respectively. For the parameter regions investigated here the main production channel is given by gluon fusion. The one-loop (leading order) computation uses FormCalc/ LoopTools [81,82]. Various cross-checks against MadGraph [83] and other results [5,84-86] have been carried out. We do not include $b$ quark loops throughout as they are negligible for the parameter regions studied in this work. ${ }^{5}$

\footnotetext{
${ }^{5}$ Specifically, for the T2 scenario we always observe $\tan \beta \sim 1$ while for $\mathrm{T} 1$ most points show $\tan \beta \simeq 7$.
} 


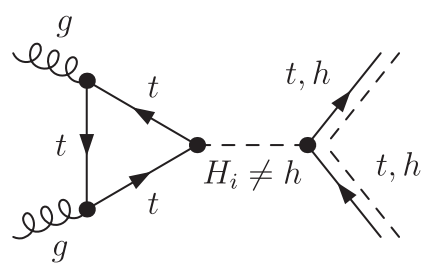

FIG. 1. Representative signal diagram contributing to $t \bar{t}$ and $h h$ resonance searches. $h$ denotes the light SM-like state with $m_{h} \simeq 125 \mathrm{GeV}$, while $H_{i}$ denotes the remaining heavy Higgs bosons that arise in the C2HDM and CxSM.

We select one state $H_{i}$, defined as the signal, and compute the squared amplitude for the $g g \rightarrow H_{i} \rightarrow t \bar{t} / h h$ process:

$$
d \sigma_{i}^{\mathrm{os}} \sim\left|\mathcal{M}_{\mathrm{sig}}\left(g g \rightarrow H_{i} \rightarrow X \bar{X}\right)\right|^{2}, \quad X=t, h,
$$

where $\mathcal{M}$ is the signal amplitude given by the $s$-channel one-loop diagrams shown in Fig. 1. This cross section can be understood as the on-shell cross section that one would obtain from $\sigma$-times-branching ratio estimates. To obtain these cross sections and put them in relation to interference effects, we integrate the cross sections within

$$
\left|m(t \bar{t} / h h)-m_{H_{i}}\right|<2 \Gamma_{H_{i}} .
$$

We keep track of the interference effects with the SM "background" and beyond the Standard Model (BSM) signal. The former is given by continuum $g g \rightarrow t \bar{t}$ production, Fig. 2, for the $t \bar{t}$ final state, and by box, Fig. 2, and off-shell $h$-induced $g g \rightarrow h h$ contributions for the $h h$ final state. The latter derives from the competing $g g \rightarrow H_{j \neq i} \rightarrow$ $h h$ diagrams (Fig. 1). This gives rise to an estimate of the observed cross section in the presence of interference effects:

$$
\begin{aligned}
d \sigma_{i} \sim & \left|\mathcal{M}_{\mathrm{sig}}\left(g g \rightarrow H_{i} \rightarrow X \bar{X}\right)\right|^{2} \\
& +2 \operatorname{Re}\left\{\mathcal{M}_{\mathrm{sig}} \mathcal{M}_{\mathrm{bkg}}^{*}\left(H_{j \neq i}, \text { cont. }\right)\right\},
\end{aligned}
$$

where "cont." stands for the continuum $t \bar{t}$ or $h h$ "background" and (off-shell) $H_{j \neq i}$ contributions as mentioned above, including the SM-like $h$.
The scans described in the previous section show that there are viable parameter choices with the tendency to produce quasidegenerate mass spectra in the C2HDM when both $t \bar{t}$ and $h h$ decay channels are open. We define the two non-SM states as "degenerate" when their mass splitting is less than $10 \%$ of the heavy scalar's mass. This accounts for most of the parameter points that are described in Sec. II.

For parameter points that have very small cross sections in either of the two channels, interference effects when considered in relation to the on-shell signal definition can be very large; however in this case they have little phenomenological importance. We therefore filter our results with some minimum cross section requirements for both $p p \rightarrow t \bar{t}$ and $p p \rightarrow h h$. For $p p \rightarrow t \bar{t}$ we require at least $170 \mathrm{fb}$ before the inclusion of $K$ factors; for $p p \rightarrow h h$ we demand at least $8 \mathrm{fb}$. This amounts to about $\mathcal{O}(0.5 \mathrm{pb})$ $[87,88]$ when higher-order corrections are included for $t \bar{t}$ final states and $\simeq 16 \mathrm{fb}$ for $h h$ production [89-96].

\section{B. Results and discussion}

\section{The C2HDM}

In order to investigate the effects from interferences for the $h h$ and $t \bar{t}$ final states, we introduce the ratio of the signal plus interference cross section $\sigma$ [defined in Eq. (21)] and the signal cross section $\sigma^{\text {os }}$ [defined in Eq. (19) for the requirement Eq. (20)], i.e.,

$$
R(x x)=\frac{\sigma(x x)}{\sigma^{\mathrm{os}}(x x)}, \quad x x=h h, t \bar{t} .
$$

In Fig. 3(a) we show $R(h h)$ versus $R(t \bar{t})$ for the C2HDM type 1 for degenerate non-SM-like Higgs states, i.e., states whose masses differ by less than $10 \%$. As can be inferred from the figure, there is a broad range of possible phenomenological outcomes. We can have a large enhancement or suppression of the $H_{i} \rightarrow t \bar{t}$ signal while the $h h$ rate can be either enhanced or reduced. Points with large constructive interference effects in the $t \bar{t}$ final state are likely to be constrained through $p p \rightarrow t \bar{t}$ measurements. We also obtain parameter points for which interference effects decrease the search potential in both the $t \bar{t}$ and $h h$ channels. Having simultaneous contributions from

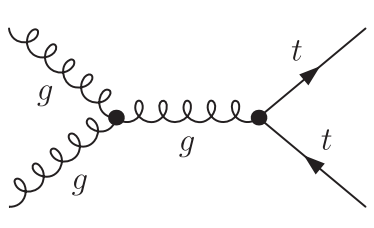

(a)

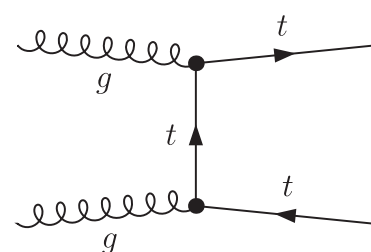

(b)

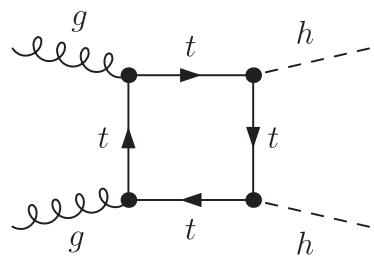

(c)

FIG. 2. Representative nonresonant "background" diagrams contributing to $p p \rightarrow t \bar{t}$ (a),(b) and $p p \rightarrow h h$ (c) searches (different fermion flows are understood implicitly). The off-shell $h$-induced background contribution derives from graphs shown in Fig. 1 with an off-shell $h$ running in the $s$-channel. 


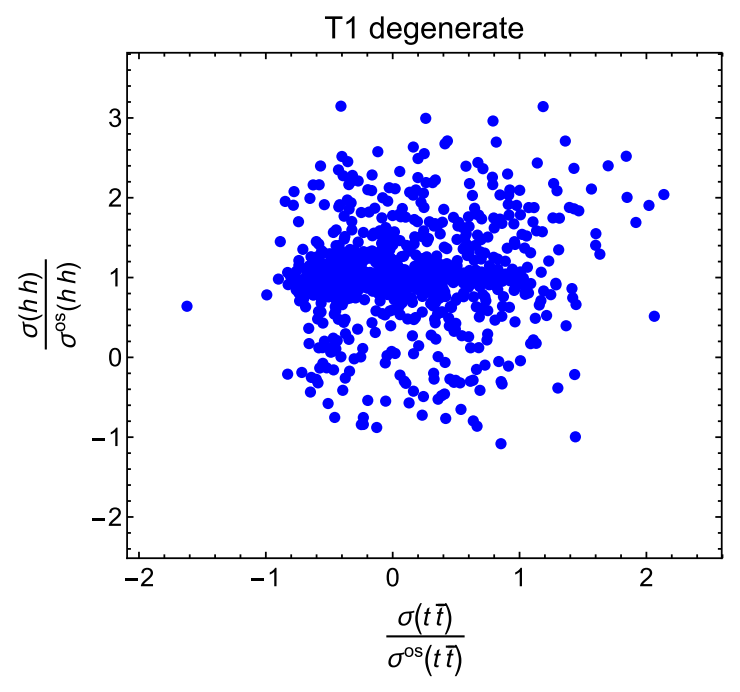

(a)

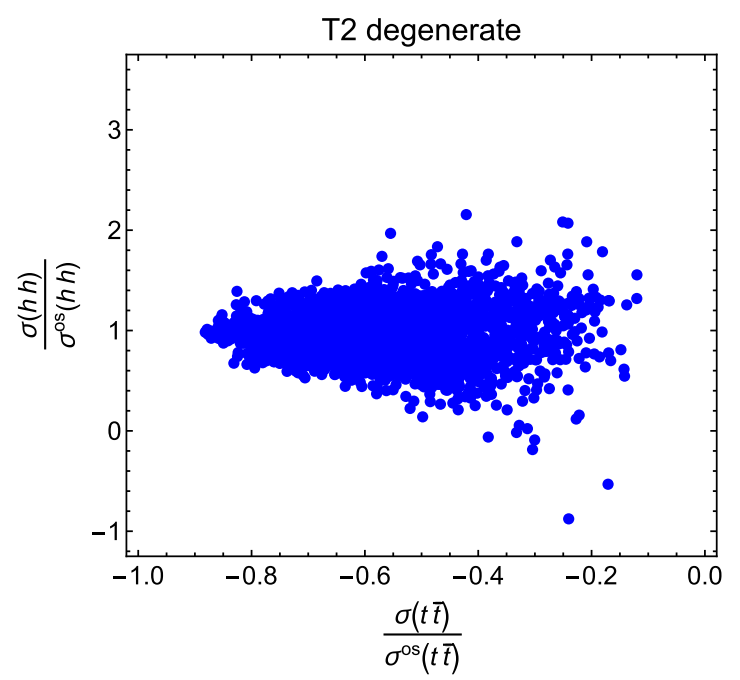

(b)

FIG. 3. Ratio of signal + interference cross section $\sigma$ and on-shell (OS) cross section $\sigma^{\text {os }}$ (for definition, see text) in $p p \rightarrow h h$ and $p p \rightarrow t \bar{t}$ for degenerate non-SM-like Higgs states. Points are preselected to have resonance cross sections of at least $170 \mathrm{fb}$ at leading order (LO) in the $t \bar{t}$ and $8 \mathrm{fb}$ in the $h h$ channels. (a) 2HDM type 1; (b) 2HDM type 2.

signal-signal (i.e., interference between the two $s$-channel $H_{i} \neq h$ contributions) and signal-background interference for the resonance masses not too far away from each other, both effects contribute when we obtain a simultaneous enhancement in the $t \bar{t}$ and $h h$ rates. Points that show this correlation are clustered around the $t \bar{t}$ threshold, $m_{H_{i}} \simeq 350 \mathrm{GeV}$, with small widths $\Gamma_{H_{i}} / m_{H_{i}} \simeq 10^{-3}$; in this region interference effects with background contributions are particularly large. As this effect tends to be destructive above the resonance mass (see also [20] or below), widening the mass range that we use to define the signal Eq. (20) can decrease the $\sigma(t \bar{t}) / \sigma^{\mathrm{os}}(t \bar{t})$ value. This can be relevant when the mass resolution is imperfect in the analyses and should be kept track of in the experimental investigation. Above the threshold, when $H_{i} \rightarrow t \bar{t}$ is kinematically accessible, so is $H_{i} \rightarrow h h$. The enhancement in $h h$ is then a combination of signal-background (see also [24]) and signal-signal interference, with the latter playing the dominant role for the parameter choices studied here.

Particularly interesting from a double Higgs discovery perspective, however, is the tendency of the $H_{i} \rightarrow t \bar{t}$ signals to be reduced, with large constructive interference effects present in $H_{i} \rightarrow h h$. As the box-graph "backgrounds" in the $p p \rightarrow h h$ case decrease with the center-of-mass energy, these effects are predominantly due to signal-signal interference for overlapping Breit-Wigner distributions when the mass spectra are quasidegenerate. This is a key implication of the scan as detailed in Sec. II: while signal-background interference decreases the sensitivity in the $t \bar{t}$ channels, which are known to be the most constraining channels in C2HDM sensitivity extrapolations (see e.g., [2]), these effects can be correlated with large signal-signal interference effects in the di-Higgs modes. Not only is the $\sigma$-times-branching ratio estimate typically inadequate in both decay modes, but di-Higgs final states can become dominant search modes for new physics in the context of the 2HDMs as discussed above.

This point is further highlighted when we consider the C2HDM of type 2 in Fig. 3(b). ${ }^{6}$ Here we always find a decrease of the expected leading-order rate for the $t \bar{t}$ spectrum. While this can be partly compensated for via large QCD corrections for the signal component, ${ }^{7}$ it is clear that the straightforward $\sigma$-times-branching ratio approximation will overestimate the sensitivity dramatically. Again different outcomes in the correlation between $h h$ and $t \bar{t}$ final states are possible: signal-signal interference in $H_{i} \rightarrow$ $h h$ can enhance or decrease the cross section expectation. Note, however, that in our scan the points with $\sigma(t \bar{t}) / \sigma^{\mathrm{os}}(t \bar{t}) \rightarrow-1$ merge towards $\sigma(h h) / \sigma^{\mathrm{os}}(h h) \rightarrow 1$.

The reason for this behavior becomes apparent from Fig. 4. On the one hand, signal-background interference in the $t \bar{t}$ mass spectrum remains large due to the large continuum background contribution even for heavy resonances of around $800 \mathrm{GeV}$. On the other hand, hierarchies in the trilinear Higgs self-couplings are directly reflected in different resonance cross sections, which implies that $p p \rightarrow$ $H_{2} \rightarrow h h$ is much more suppressed than $p p \rightarrow H_{3} \rightarrow h h$, which also implies that signal-signal interference is not relevant for the $\mathrm{H}_{3} \rightarrow$ hh decay with a phenomenologically

\footnotetext{
${ }^{6}$ As detailed in Ref. [97], an additional requirement on the charged Higgs mass $m_{H^{ \pm}}>590 \mathrm{GeV}$ for type 1 (for type 2 $m_{H^{ \pm}}>590 \mathrm{GeV}$ is enforced by the constraint from the weak radiative $B$ meson decays [70]) leads to qualitative agreement between the type 1 and type 2 models.

${ }^{7}$ Note that as we typically deal with small width values, issues that relate to the precise definition of signal and background $[98,99]$ are numerically suppressed and $\sigma$-times-branching ratio extrapolations would be justified if there was no interference.
} 


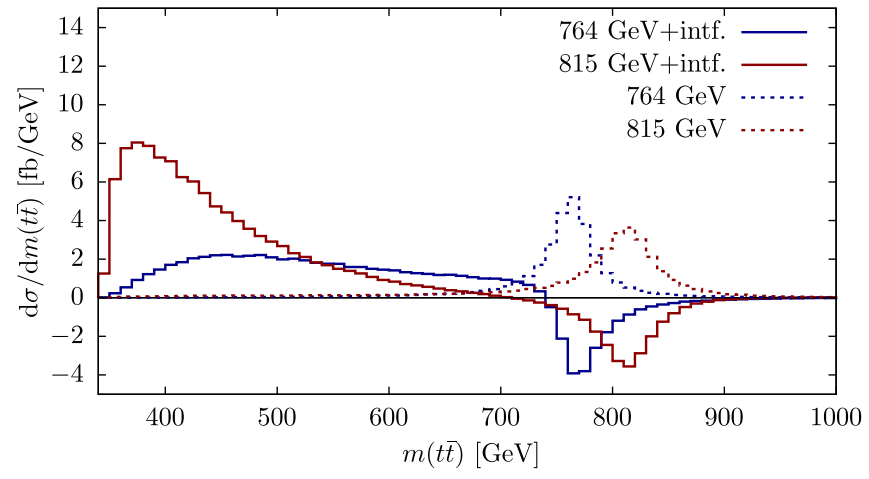

(a)

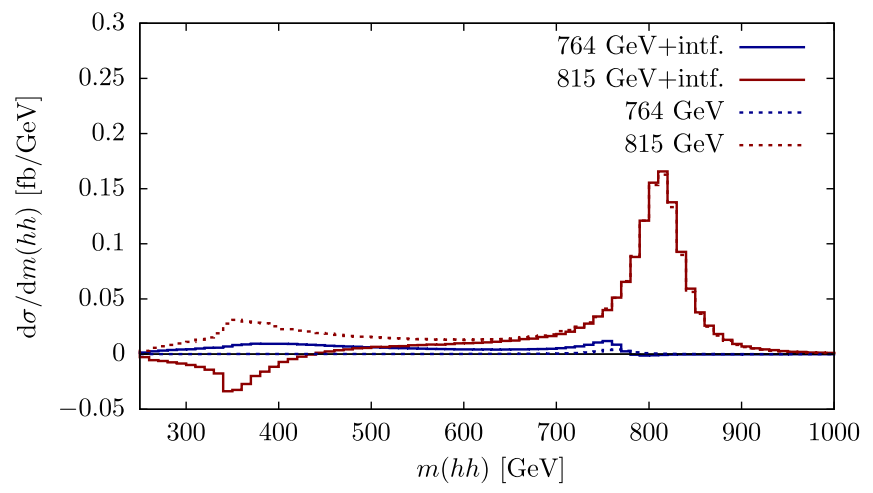

(b)

FIG. 4. Comparison of the invariant mass distribution for $p p \rightarrow t \bar{t}$ (a) and $p p \rightarrow h h$ (b) at $13 \mathrm{TeV}$ at $\mathrm{LO}$ for the different states $H_{i} \neq h$ (blue, $H_{i}=H_{2}$; red, $H_{i}=H_{3}$ ). We show the signal $g g \rightarrow t \bar{t}$ and $g g \rightarrow h h$ production following Eq. (19) as dashed lines. The interference-corrected cross sections, Eq. (21), are depicted as solid lines. The spectra arise from the parameter point BP1; see Table III.

significant cross section. For the particular benchmark point BPI (see Table III), that we choose to illustrate this effect in Fig. 4, we find a large signal-background interference such that we end up with a dip structure in $t \bar{t}$ with about $\simeq-200 \mathrm{fb}$ when integrating Eq. (21) over the range of Eq. (20).

TABLE III. Parameter points as shown in Figs. 4-7. For details of the calculation see Sec. II D. All points are not constrained by the most recent CMS analysis of Ref. [22]. Note that for the plots we used LO rates. The benchmarks reflect the experimental status as summarized in the tool chain detailed in Sec. II.

\begin{tabular}{lcccc}
\hline \hline & BP1 & BP2 & BP3 & BP4 \\
\hline$m_{H_{1}}(\mathrm{GeV})$ & 125.090 & 125.090 & 125.090 & 125.090 \\
$m_{H_{2}}(\mathrm{GeV})$ & 764.044 & 691.319 & 608.588 & 442.903 \\
$m_{H_{3}}(\mathrm{GeV})$ & 814.578 & 694.637 & 609.393 & 626.371 \\
$m_{H^{ \pm}}(\mathrm{GeV})$ & 853.064 & 654.204 & 679.601 & 651.550 \\
$\alpha_{1}$ & 0.746 & 0.766 & 0.818 & 0.736 \\
$\alpha_{2}$ & -0.132 & 0.042 & 0.053 & 0.045 \\
$\alpha_{3}$ & -0.086 & 1.144 & 0.913 & 1.567 \\
$\tan (\beta)$ & 0.921 & 0.870 & 0.892 & 0.928 \\
$R_{13}^{2}$ & 0.017 & 0.002 & 0.003 & 0.002 \\
$R_{23}^{2}$ & 0.007 & 0.827 & 0.624 & 0.998 \\
$R_{33}^{2}$ & 0.975 & 0.171 & 0.373 & 0.000 \\
$\sigma\left(g g \rightarrow H_{1}\right)(\mathrm{pb})$ & 45.908 & 49.699 & 53.640 & 43.233 \\
$\sigma\left(g g \rightarrow H_{2}\right)(\mathrm{pb})$ & 0.651 & 1.700 & 2.903 & 19.042 \\
$\sigma\left(g g \rightarrow H_{3}\right)(\mathrm{pb})$ & 0.637 & 1.284 & 2.670 & 1.899 \\
$\lambda_{H_{1} H_{1} H_{1}}(\mathrm{GeV})$ & -30.633 & 150.815 & 115.626 & -184.173 \\
$\lambda_{H_{1} H_{1} H_{2}}(\mathrm{GeV})$ & -49.478 & 253.524 & 305.386 & -55.652 \\
$\lambda_{H_{1} H_{1} H_{3}}(\mathrm{GeV})$ & -448.381 & 120.882 & -121.714 & 6.123 \\
$\Gamma\left(H_{1}\right)(\mathrm{GeV})$ & 0.004 & 0.004 & 0.004 & 0.004 \\
$\Gamma\left(H_{2}\right)(\mathrm{GeV})$ & 36.623 & 41.150 & 31.551 & 21.580 \\
$\Gamma\left(H_{3}\right)(\mathrm{GeV})$ & 51.865 & 34.787 & 29.057 & 32.449 \\
$\mathrm{BR}\left(H_{2} \rightarrow H_{1} H_{1}\right)$ & 0.001 & 0.021 & 0.044 & 0.003 \\
$\mathrm{BR}\left(H_{2} \rightarrow t \bar{t}\right)$ & 0.936 & 0.962 & 0.922 & 0.990 \\
$\mathrm{BR}\left(H_{3} \rightarrow H_{1} H_{1}\right)$ & 0.045 & 0.006 & 0.008 & 0.000 \\
$\mathrm{BR}\left(H_{3} \rightarrow t \bar{t}\right)$ & 0.871 & 0.979 & 0.965 & 0.793 \\
\hline \hline
\end{tabular}

Particularly interesting are again parameter points for which $t \bar{t}$ is decreased relative to the on-shell expectation while $h h$ is enhanced. An example of such an outcome is BP2 (see Table III). As can be inferred from Fig. 5, we find a large signal-background interference in $t \bar{t}$ at LO, leading to a suppression of $-420 \mathrm{fb}$, while the $h h$ signal with mass $m_{H_{3}}=694 \mathrm{GeV}$ is enhanced by a factor of 2.3 due to signal-signal interference. Also for this benchmark point phenomenologically more relevant resonant production of $\mathrm{H}_{2}$ with a mass of $691 \mathrm{GeV}$ is slightly enhanced due to signal-signal interference. Analogous to our results for C2HDM type 1, this indicates that usual SM search channels might have suppressed sensitivity and new physics could indeed be observed in di-Higgs production first. Although the $h h$ cross section is significantly smaller compared to the $t \bar{t}$ rate, the relative enhancement through signal-signal interference magnifies the importance of di-Higgs searches at the LHC. Another, milder example along this line is given by BP3 (Fig. 6) where the $t \bar{t}$ resonance structure is significantly distorted to a peak-dip combination, while $p p \rightarrow h h$ remains as a Breit-Wigner peak at a slight $30 \%$ suppression (LO) rate. The masses of $\mathrm{H}_{2}$ and $\mathrm{H}_{3}$ in Figs. 5 and 6 are quite similar, and indeed the curves on the left-hand sides of the figures $(t \bar{t})$ are quite similar for the two masses. It is interesting, however, that the difference between the curves for these two mass points on the right-hand sides of the figures $(h h)$ is clearly visible, due to the difference in the signal-signal interference that can be observed in $p p \rightarrow h h$ production.

The qualitative behavior that we have found so far is related to the fact that the scalar spectra are quite compressed in the examples we have considered so far, where we have

$$
\left|m_{H_{2}}-m_{H_{3}}\right|<0.1 m_{H_{3}} .
$$

Given the finite experimental resolution, states that are nearly degenerate are difficult to resolve. Nonetheless, if an 


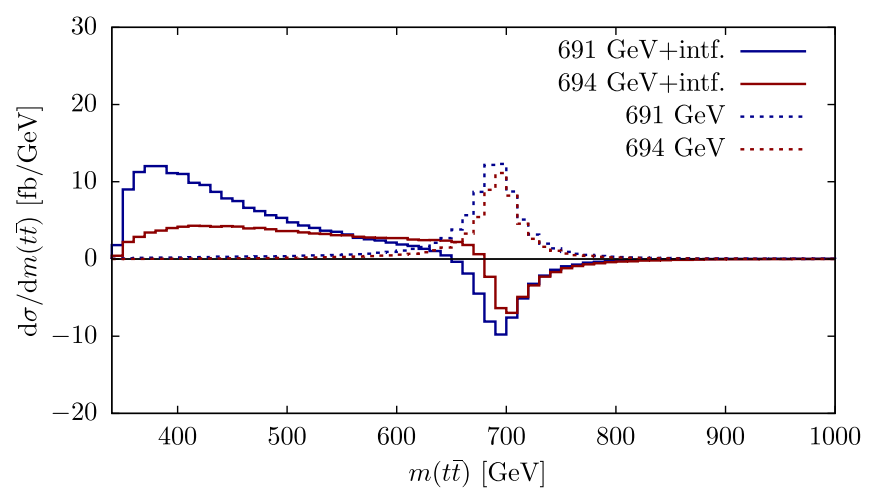

(a)

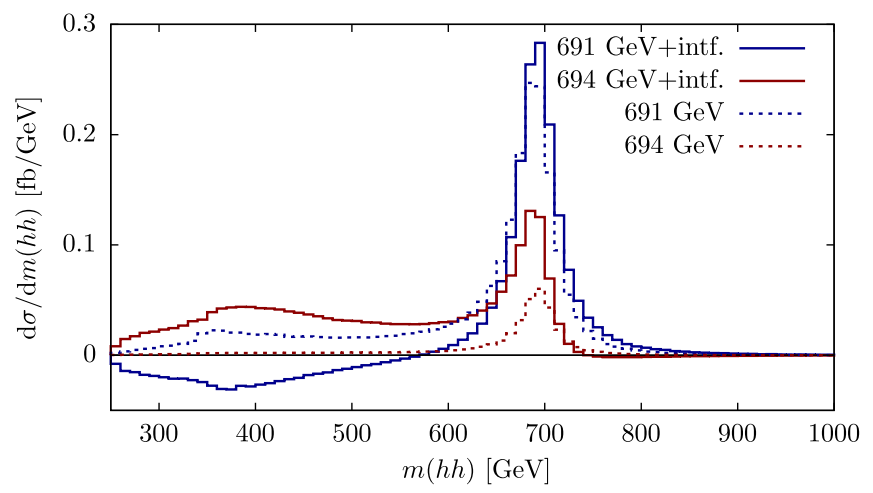

(b)

FIG. 5. Comparison of the invariant mass distribution for $p p \rightarrow t \bar{t}$ (a) and $p p \rightarrow h h$ (b) at $13 \mathrm{TeV}$ at LO for the different states $H_{i} \neq h$ (blue: $H_{i}=H_{2}$, red: $H_{i}=H_{3}$ ). We show the signal $g g \rightarrow t \bar{t}$ and $g g \rightarrow h h$ production following Eq. (19) as dashed lines. The interference-corrected cross sections, Eq. (21), are depicted as solid lines. The spectra arise from the parameter point BP2; see Table III.

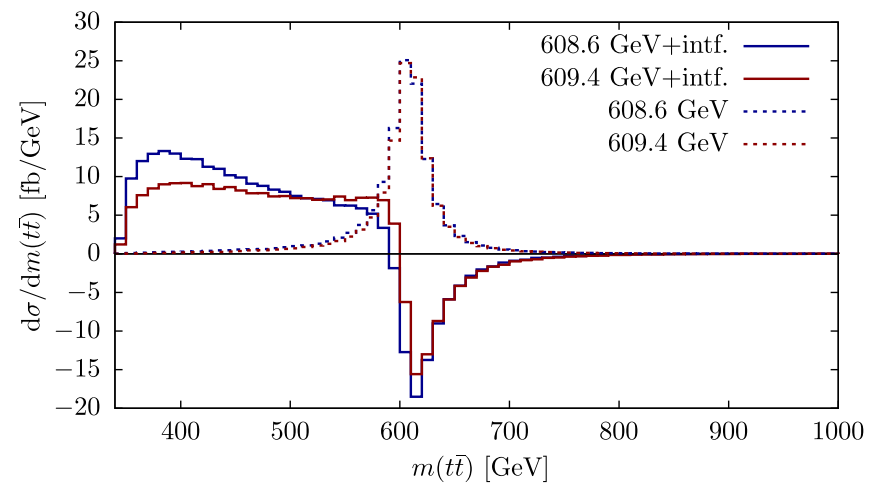

(a)

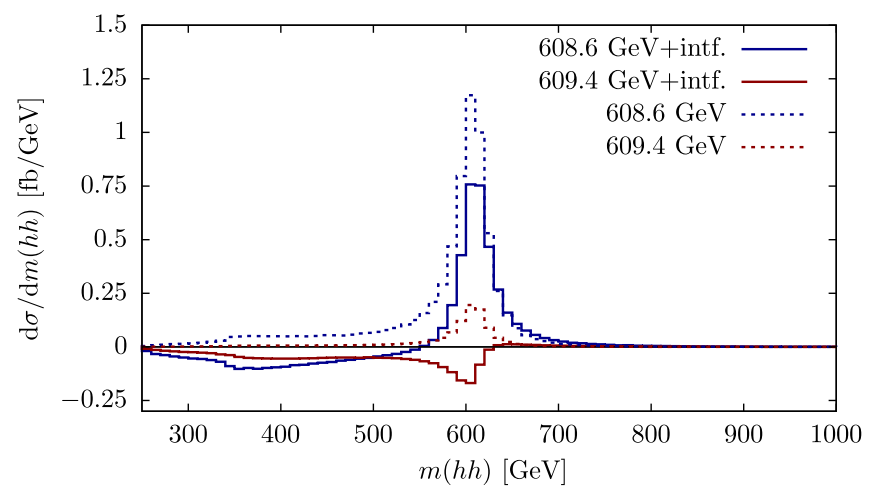

(b)

FIG. 6. Comparison of the invariant mass distribution for $p p \rightarrow t \bar{t}$ (a) and $p p \rightarrow h h$ (b) at $13 \mathrm{TeV}$ at LO for the different states $H_{i} \neq h$ (blue, $H_{i}=H_{2}$; red, $H_{i}=H_{3}$ ). We show the signal $g g \rightarrow t \bar{t}$ and $g g \rightarrow h h$ production following Eq. (19) as dashed lines. The interference-corrected cross sections, Eq. (21), are depicted as solid lines. The spectra arise from the parameter point BP3; see Table III.

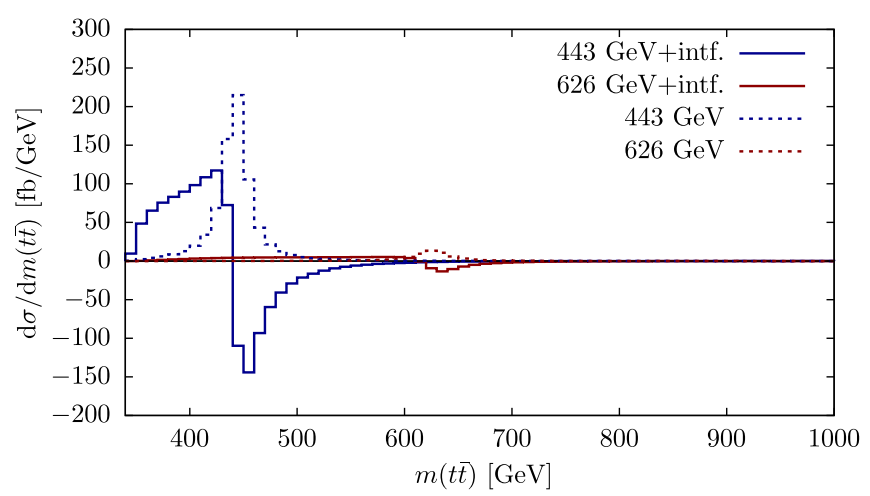

(a)

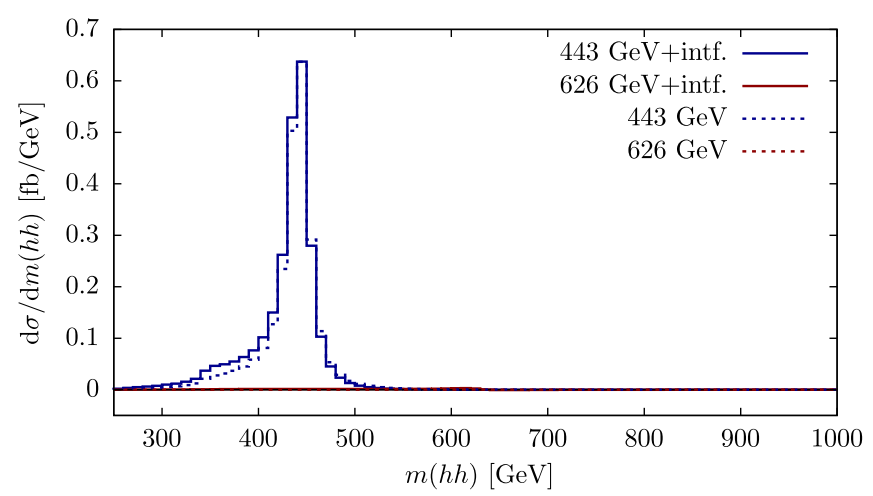

(b)

FIG. 7. Comparison of the invariant mass distribution for $p p \rightarrow t \bar{t}$ (a) and $p p \rightarrow h h$ (b) at $13 \mathrm{TeV}$ at LO for the different states $H_{i} \neq h$ (blue, $H_{i}=H_{2}$; red, $H_{i}=H_{3}$ ). We show the signal $g g \rightarrow t \bar{t}$ and $g g \rightarrow h h$ production following Eq. (19) as dashed lines. The interference-corrected cross sections, Eq. (21), are depicted as solid lines. The spectra arise from the parameter point BP4; see Table III. 


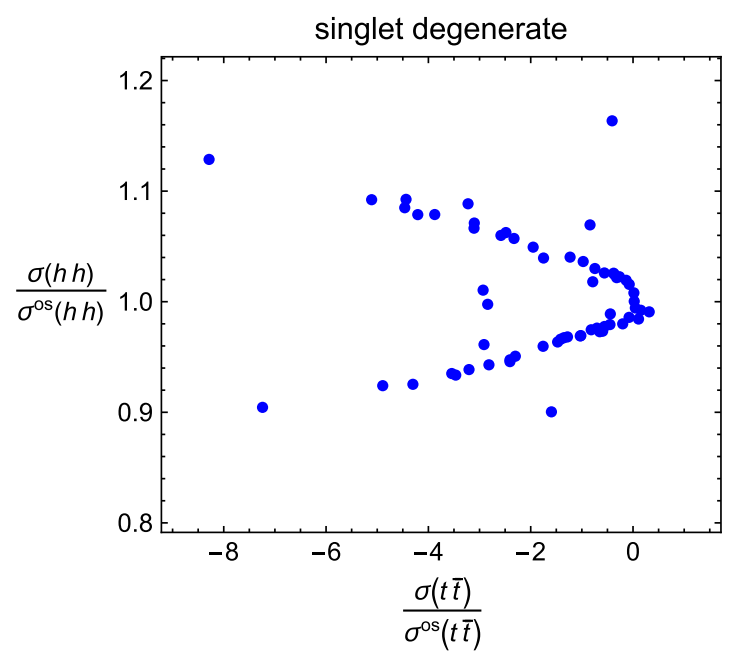

(a)

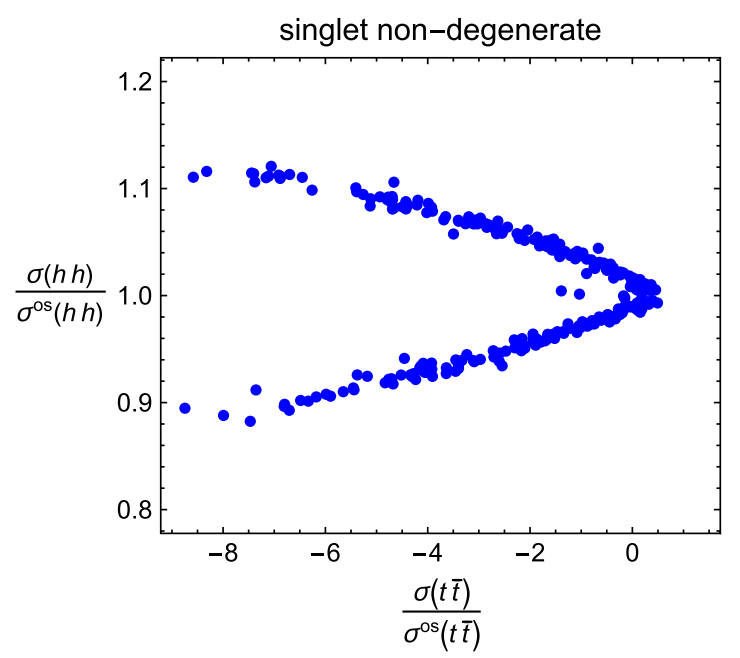

(b)

FIG. 8. Ratio of signal + interference cross section $\sigma$ and OS cross section $\sigma^{\mathrm{os}}$ in $p p \rightarrow h h$ and $p p \rightarrow t \bar{t}$ in the CxSM scenario. Points are preselected to have at least $8 \mathrm{fb}$ in the $h h$ channels at leading order. Due to the nature of this model the absolute values of the $t \bar{t}$ final state rates are phenomenologically irrelevant as they are $\mathcal{O}(\mathrm{fb})$. For details see text. (a) Degenerate and (b) nondegenerate non-SM-like Higgs bosons.

excess is observed in the future, signal-signal interference is an important effect that needs to be taken into account if the excess is interpreted along the lines of two nearly degenerate states in a C2HDM.

It is also interesting to discuss the opposite situation when the two scalars lie further apart in mass. Under these circumstances, signal-signal interference is largely absent in $h h$ production, while signal-background interference again distorts or even removes the resonance structure in $H_{i} \rightarrow t \bar{t}$. When $\left|m_{H_{2}}-m_{H_{3}}\right|>0.1 m_{H_{3}}$ we can therefore conclude that $\sigma$-times-branching ratio estimates provide an accurate description of the $h h$ phenomenology while the sensitivity in $t \bar{t}$ is again overestimated, as illustrated in Fig. 7. For this point BP4 (see Table III), we again observe a large signal-background interference leading to $\simeq-300 \mathrm{fb}$ for the on-shell region according to Eq. (20) in $t \bar{t}$ with $p p \rightarrow h h$ production remaining stable.

\section{The CxSM}

We finally turn to the relevance of $C P$ violation in the above context. To this end, it is helpful to consider the $\mathrm{CxSM}$ of Sec. II A. Again, a wide range of mass spectra can be obtained that are consistent with the scan outlined in Sec. II B. This includes compressed spectra according to Eq. (23) through selecting appropriate parameters of the extended Higgs potential that pass LHC constraints.

As we are dealing with a model that anticorrelates SM Higgs coupling consistency with the size of the exotic scalar cross sections, $H_{i} \rightarrow t \bar{t}$ production is typically not phenomenologically relevant. This is also the reason why interference effects can be very large in this case as the signal cross section can be negligible. Nonetheless it is interesting to observe that the di-Higgs cross section is not influenced dramatically and qualitatively identical when we compare degenerate and nondegenerate mass spectra in Fig. 8. All points are around $m_{H_{i}} \simeq 400 \mathrm{GeV}$ where the SM di-Higgs cross section reaches a maximum. The second non-SM-like Higgs boson $H_{j}$ has either a mass close to $m_{H_{i}}$ according to our criteria Eq. (23) (left plot) or is far apart (right plot). In this scenario we deal indeed with only mild signal-background and signal-signal interference; i.e., $\sigma$-times-branching ratio expectations provide an accurate estimate of the $h h$ phenomenology in this scenario. This can be contrasted with the C2HDM phenomenology: when we switch on $C P$ violation, we typically create the possibility of gauge-phobic Higgs bosons (we remind the reader that in the CxSM we only deal with neutral $C P$-even Higgs bosons), which then preferably couple to top quarks when the gluon fusion production mode is large. Additionally, when the trilinear couplings are large enough, $H_{i} \rightarrow h h$ can become sizable while $H_{i} \rightarrow t \bar{t}$ is opening up to large signal-background interference effects.

\section{CONCLUSIONS}

Signal-background interference is a phenomenologically important feature in resonance searches in top final states. In this paper we have shown that the signal-background interference that the ATLAS and CMS experiments are already considering in setting limits on models of the kind discussed in this work needs to be extended to signalsignal interference effects, in particular when considering di-Higgs production as a BSM discovery tool.

While constructive enhancements in the $t \bar{t}$ final states with large cross sections could be a smoking gun of BSM 
physics in the near future (see the recent [18]), an equally interesting outcome is the decrease of the $t \bar{t}$ signal due to signal-background interference that is correlated with an enhancement of the resonant di-Higgs production rate as a consequence of significant signal-signal interference. The latter becomes important when extra scalar resonances are heavy and reasonably close in mass. This is a region of the C2HDM parameter space which naturally arises given current LHC observations. Our findings therefore indicate that the relevance of di-Higgs final states in the context of the $C P$-violating $\mathrm{C} 2 \mathrm{HDM}$ has so far been underestimated. We provide a number of benchmark scenarios that highlight the phenomenological relation of $t \bar{t}$ and $h h$ resonance searches. We limit ourselves to stable top and Higgs boson final states in this work. $t \bar{t}$ resonance searches are well under way (e.g., [22]), and the large SM backgrounds that can be limiting factors of di-Higgs searches have been shown to be manageable; see the recent CMS analysis of Ref. [100].

In actual experimental analyses, the discrimination of nearly degenerate resonances is hampered by the finite resolution that can be obtained in $t \bar{t}$ or di-Higgs resonance searches. Yet, interference effects are important to reach to correct microscopic parameter interpretation.

It is worthwhile to note that similar effects are not present in simpler scenarios such as, e.g., a complex scalar portal extension of the Higgs sector. In the mass region where the effects discussed in this paper work efficiently, the cross sections in $t \bar{t}$ are too suppressed to be phenomenologically relevant, which de facto decorrelates the $t \bar{t}$ and $h h$ channels. In the CxSM the $t \bar{t}$ channels are suppressed (see also [31]) while in the C2HM interference effects distort the shapes of the resonances. While the latter makes discoveries in the $t \bar{t}$ channel difficult the discovery of a new scalar resonance with a significant cross section in $t \bar{t}$ would strongly discriminate between the C2HDM and the CxSM.

\section{ACKNOWLEDGMENTS}

P. B. acknowledges financial support by the Graduiertenkolleg GRK 1694: Elementarteilchenphysik bei höchster Energie und höchster Präzision. M. M. acknowledges support by the Deutsche Forschungsgemeinschaft (DFG, German Research Foundation) under Grant No. 396021762-TRR 257. S. D. is supported by the U.S. Department of Energy under Grant No. de-sc0012704. C. E. is supported by the UK Science and Technology Facilities Council (STFC) under Grant No. ST/P000746/1. This work was supported by the Munich Institute for Astro- and Particle Physics (MIAPP) of the DFG Excellence Cluster Origins (www.origins-cluster.de).
[1] E. Bagnaschi, Eur. Phys. J. C 79, 617 (2019)

[2] P. Basler, S. Dawson, C. Englert, and M. Muhlleitner, Phys. Rev. D 99, 055048 (2019).

[3] S. Baum and N. R. Shah, arXiv:1904.10810.

[4] K. S. Babu and S. Jana, J. High Energy Phys. 02 (2019) 193.

[5] S. Dittmaier et al. (LHC Higgs Cross Section Working Group), arXiv:1101.0593.

[6] K. J. F. Gaemers and F. Hoogeveen, Phys. Lett. 146B, 347 (1984).

[7] D. Dicus, A. Stange, and S. Willenbrock, Phys. Lett. B 333, 126 (1994).

[8] W. Bernreuther, A. Brandenburg, and M. Flesch, arXiv: hep-ph/9812387.

[9] S. Jung, J. Song, and Y. W. Yoon, Phys. Rev. D 92, 055009 (2015).

[10] R. Frederix and F. Maltoni, J. High Energy Phys. 01 (2009) 047.

[11] V. Barger, T. Han, and D. G. E. Walker, Phys. Rev. Lett. 100, 031801 (2008).

[12] N. Craig, F. D'Eramo, P. Draper, S. Thomas, and H. Zhang, J. High Energy Phys. 06 (2015) 137.

[13] W. Bernreuther, P. Galler, C. Mellein, Z. G. Si, and P. Uwer, Phys. Rev. D 93, 034032 (2016).

[14] M. Carena and Z. Liu, J. High Energy Phys. 11 (2016) 159.
[15] B. Hespel, F. Maltoni, and E. Vryonidou, J. High Energy Phys. 10 (2016) 016.

[16] D. Buarque Franzosi, F. Fabbri, and S. Schumann, J. High Energy Phys. 03 (2018) 022.

[17] D. Buarque Franzosi, E. Vryonidou, and C. Zhang, J. High Energy Phys. 10 (2017) 096.

[18] A. Djouadi, J. Ellis, A. Popov, and J. Quevillon, J. High Energy Phys. 03 (2019) 119.

[19] N. Kauer, A. Lind, P. Maierhöfer, and W. Song, J. High Energy Phys. 07 (2019) 108.

[20] M. Aaboud et al. (ATLAS Collaboration), Phys. Rev. Lett. 119, 191803 (2017).

[21] G. Brooijmans et al., J. High Energy Phys. 03 (2018) 178, http://lss.fnal.gov/archive/2017/conf/fermilab-conf17-664-ppd.pdf.

[22] A. M. Sirunyan et al. (CMS Collaboration), arXiv:1908 .01115 .

[23] J. Alison et al., in Double Higgs Production, Batavia, edited by B. Di Micco, M. Gouzevitch, J. Mazzitelli, and C. Vernieri (FermiLab/CERN, 2019), https://lss.fnal.gov/ archive/2019/conf/fermilab-conf-19-468-e-t.pdf.

[24] S. Dawson and I. M. Lewis, Phys. Rev. D 92, 094023 (2015).

[25] V. Barger, P. Langacker, M. McCaskey, M. RamseyMusolf, and G. Shaughnessy, Phys. Rev. D 79, 015018 (2009). 
[26] M. Gonderinger, H. Lim, and M. J. Ramsey-Musolf, Phys. Rev. D 86, 043511 (2012).

[27] R. Coimbra, M. O. P. Sampaio, and R. Santos, Eur. Phys. J. C 73, 2428 (2013).

[28] M. Jiang, L. Bian, W. Huang, and J. Shu, Phys. Rev. D 93, 065032 (2016).

[29] F. Sannino and J. Virkajärvi, Phys. Rev. D 92, 045015 (2015).

[30] R. Costa, M. Mühlleitner, M. O. P. Sampaio, and R. Santos, J. High Energy Phys. 06 (2016) 034.

[31] M. Mühlleitner, M. O. P. Sampaio, R. Santos, and J. Wittbrodt, J. High Energy Phys. 08 (2017) 132.

[32] C.-W. Chiang, M. J. Ramsey-Musolf, and E. Senaha, Phys. Rev. D 97, 015005 (2018).

[33] D. Azevedo, P. Ferreira, M. M. Mühlleitner, R. Santos, and J. Wittbrodt, Phys. Rev. D 99, 055013 (2019).

[34] P. M. Ferreira, R. Guedes, M. O. P. Sampaio, and R. Santos, J. High Energy Phys. 12 (2014) 067.

[35] G. Aad et al. (ATLAS, CMS Collaborations), Phys. Rev. Lett. 114, 191803 (2015).

[36] M. E. Peskin and T. Takeuchi, Phys. Rev. D 46, 381 (1992).

[37] I. Maksymyk, C. P. Burgess, and D. London, Phys. Rev. D 50, 529 (1994).

[38] R. Costa, A. P. Morais, M. O. P. Sampaio, and R. Santos, Phys. Rev. D 92, 025024 (2015).

[39] P. Bechtle, O. Brein, S. Heinemeyer, G. Weiglein, and K. E. Williams, Comput. Phys. Commun. 181, 138 (2010).

[40] P. Bechtle, O. Brein, S. Heinemeyer, G. Weiglein, and K. E. Williams, Comput. Phys. Commun. 182, 2605 (2011).

[41] P. Bechtle, O. Brein, S. Heinemeyer, O. Stal, T. Stefaniak, G. Weiglein, and K. E. Williams, Eur. Phys. J. C 74, 2693 (2014).

[42] P. Bechtle, S. Heinemeyer, O. Stål, T. Stefaniak, and G. Weiglein, Eur. Phys. J. C 74, 2711 (2014).

[43] R. V. Harlander, S. Liebler, and H. Mantler, Comput. Phys. Commun. 184, 1605 (2013).

[44] R. V. Harlander, S. Liebler, and H. Mantler, Comput. Phys. Commun. 212, 239 (2017).

[45] A. Djouadi, J. Kalinowski, and M. Spira, Comput. Phys. Commun. 108, 56 (1998).

[46] A. Djouadi, J. Kalinowski, M. Muehlleitner, and M. Spira, arXiv:1801.09506.

[47] M. Tanabashi et al. (Particle Data Group), Phys. Rev. D 98, 030001 (2018).

[48] A. E. A. Denner, CERN Report No. LHCHXSWG-INT2015-006, https://cds.cern.ch/record/2047636.

[49] A. D. Sakharov, Pis'ma Zh. Eksp. Teor. Fiz. 5, 32 (1967); Usp. Fiz. Nauk 161, 61 (1991).

[50] M. Quiros, Helv. Phys. Acta 67, 451 (1994).

[51] G. D. Moore, Phys. Rev. D 59, 014503 (1998).

[52] P. Basler and M. Mühlleitner, Comput. Phys. Commun. 237, 62 (2019).

[53] T. D. Lee, Phys. Rev. D 8, 1226 (1973).

[54] J. F. Gunion, H. E. Haber, G. L. Kane, and S. Dawson, Front. Phys. 80, 1 (2000).

[55] G. C. Branco, P. M. Ferreira, L. Lavoura, M. N. Rebelo, M. Sher, and J. P. Silva, Phys. Rep. 516, 1 (2012).

[56] I. F. Ginzburg, M. Krawczyk, and P. Osland, in Linear Colliders. Proceedings LCWS 2002 (2002), pp. 703-706, http://weblib.cern.ch/abstract?CERN-TH-2002-330.
[57] D. Fontes, J. C. Romão, and J. P. Silva, J. High Energy Phys. 12 (2014) 043.

[58] L. Lavoura and J. P. Silva, Phys. Rev. D 50, 4619 (1994).

[59] F. J. Botella and J. P. Silva, Phys. Rev. D 51, 3870 (1995).

[60] A. W. El Kaffas, P. Osland, and O. M. Ogreid, Nonlinear Phenom. Complex Syst. 10, 347 (2007).

[61] D. Fontes, M. Mühlleitner, J. C. Romão, R. Santos, J. P. Silva, and J. Wittbrodt, J. High Energy Phys. 02 (2018) 073.

[62] H. E. Haber and O. Stål, Eur. Phys. J. C 75, 491 (2015); 76, 312(E) (2016).

[63] J. Baglio, O. Eberhardt, U. Nierste, and M. Wiebusch, Phys. Rev. D 90, 015008 (2014).

[64] I. P. Ivanov and J. P. Silva, Phys. Rev. D 92, 055017 (2015).

[65] H. E. Haber and H. E. Logan, Phys. Rev. D 62, 015011 (2000).

[66] O. Deschamps, S. Descotes-Genon, S. Monteil, V. Niess, S. T'Jampens, and V. Tisserand, Phys. Rev. D 82, 073012 (2010).

[67] F. Mahmoudi and O. Stal, Phys. Rev. D 81, 035016 (2010).

[68] T. Hermann, M. Misiak, and M. Steinhauser, J. High Energy Phys. 11 (2012) 036.

[69] M. Misiak et al., Phys. Rev. Lett. 114, 221801 (2015).

[70] M. Misiak and M. Steinhauser, Eur. Phys. J. C 77, 201 (2017).

[71] M. Baak, J. Cúth, J. Haller, A. Hoecker, R. Kogler, K. Mönig, M. Schott, and J. Stelzer (Gfitter Group), Eur. Phys. J. C 74, 3046 (2014).

[72] S. Dawson and M. Sullivan, Phys. Rev. D 97, 015022 (2018).

[73] S. Inoue, M. J. Ramsey-Musolf, and Y. Zhang, Phys. Rev. D 89, 115023 (2014).

[74] V. Andreev et al. (ACME Collaboration), Nature (London) 562, 355 (2018).

[75] C.-Y. Chen, S. Dawson, and Y. Zhang, J. High Energy Phys. 06 (2015) 056.

[76] P. Basler, M. Mühlleitner, and J. Wittbrodt, J. High Energy Phys. 03 (2018) 061.

[77] K. Arnold et al., Comput. Phys. Commun. 180, 1661 (2009).

[78] J. Baglio et al., arXiv:1107.4038.

[79] K. Arnold et al., arXiv:1207.4975.

[80] J. Baglio et al., arXiv:1404.3940.

[81] T. Hahn, Acta Phys. Pol. B 30, 3469 (1999).

[82] T. Hahn and C. Schappacher, Comput. Phys. Commun. 143, 54 (2002).

[83] J. Alwall, R. Frederix, S. Frixione, V. Hirschi, F. Maltoni, O. Mattelaer, H. S. Shao, T. Stelzer, P. Torrielli, and M. Zaro, J. High Energy Phys. 07 (2014) 079.

[84] M. Spira, arXiv:hep-ph/9510347.

[85] M. Spira, Nucl. Instrum. Methods Phys. Res., Sect. A 389, 357 (1997).

[86] C. Anastasiou, S. Bucherer, and Z. Kunszt, J. High Energy Phys. 10 (2009) 068.

[87] C. Anastasiou, C. Duhr, F. Dulat, E. Furlan, T. Gehrmann, F. Herzog, and B. Mistlberger, J. High Energy Phys. 03 (2015) 091.

[88] C. Anastasiou, C. Duhr, F. Dulat, E. Furlan, T. Gehrmann, F. Herzog, and B. Mistlberger, Phys. Lett. B 737, 325 (2014).

[89] S. Borowka, N. Greiner, G. Heinrich, S. Jones, M. Kerner, J. Schlenk, U. Schubert, and T. Zirke, Phys. Rev. Lett. 117, 012001 (2016); 117, 079901(E) (2016). 
[90] S. Borowka, N. Greiner, G. Heinrich, S. P. Jones, M. Kerner, J. Schlenk, and T. Zirke, J. High Energy Phys. 10 (2016) 107.

[91] G. Heinrich, S. P. Jones, M. Kerner, G. Luisoni, and E. Vryonidou, J. High Energy Phys. 08 (2017) 088.

[92] J. Baglio, F. Campanario, S. Glaus, M. Mühlleitner, M. Spira, and J. Streicher, Eur. Phys. J. C 79, 459 (2019).

[93] J. Davies, G. Mishima, M. Steinhauser, and D. Wellmann, J. High Energy Phys. 01 (2019) 176.

[94] R. Bonciani, G. Degrassi, P. P. Giardino, and R. Grober, Phys. Rev. Lett. 121, 162003 (2018).
[95] C.-Y. Chen, S. Dawson, and I. M. Lewis, Phys. Rev. D 91, 035015 (2015).

[96] R. Grober, M. Muhlleitner, and M. Spira, Nucl. Phys. B925, 1 (2017).

[97] P. Basler, P. M. Ferreira, M. Mühlleitner, and R. Santos, Phys. Rev. D 97, 095024 (2018).

[98] G. Passarino, C. Sturm, and S. Uccirati, Nucl. Phys. B834, 77 (2010).

[99] S. Goria, G. Passarino, and D. Rosco, Nucl. Phys. B864, 530 (2012).

[100] A. M. Sirunyan et al. (CMS Collaboration), Phys. Rev. Lett. 122, 121803 (2019). 